\title{
Impact of trauma and emergency surgery reorganization
}

\author{
Chandio A*, Sabri S, Abassi M, Chandio K, and Naqvi SA \\ Department of Surgery, Tameside NHS Foundation Trust Hospital, UK
}

\begin{abstract}
Injury is a major health problem worldwide, In the UK trauma is a leading cause of death, in British citizens across all age group with over 16000 deaths due to injury in England and Wales each year. In many countries trauma is unrecognised due to paucity of available statistics. For every fatality there are at least two survivors with serious permanent disabilities and half of them die before they reach any medical care. The facilities for treating the injured and their effectiveness vary in different institutions. The global burden of the disease due to trauma is expected to increase dramatically in coming years. The WHO predicts Road Traffic Accident will become second leading cause of death by 2020. After 2005 terror attack the delivery of trauma care and training of general trauma surgeons in the United Kingdom changed greatly and trauma infrastructure is rapidly evolving. There is still an urgent need for specialist trauma surgeons following this terrorist attack. Trauma is a major cause of disability and fatality, trauma victims continue to suffer and have direct impact on physical activities as well as psychological stress, chronic pain, feeling isolated, burden placed on family members and financial constraints affect overall quality life.
\end{abstract}

\section{Background}

Trauma is the fourth leading cause of death in the western world it is the fourth leading cause of death in the first four decades of life [1] and Trauma will become leading cause of death by 2020 [2]. The need for specialist care for the injured, in order to reduce mortality and disability was recognized by the UK government as early as 1936 [3]. The opening of Birmingham accident hospital in 1942 was a further step towards trauma care in UK. Anderson, Woodford, Dombal, Irving conducted a retrospective study of 1000 death from injury in England and Wales the study concluded that preventable trauma death over 33\% in England and Wales, the study recommended need for change in trauma-related services [4]. The same results are reflected in British Orthopaedic Association report BOA [5], this study reviewed 263 hospitals in UK and identified deficiencies in staff and equipment, many units were so small to sustain an adequate standard of care, and the report recommended need for a regional trauma centre with its multidisciplinary arrangement, rapid transfer for suitable hospital and direct senior involvement. In a retrospective study conducted as preliminary analysis of the care of injured patient in 33 British hospitals which is the first reported Trauma study in United Kingdom. Major trauma outcome study (MTOS) reported by Yates et al. [6] concluded that initial resuscitation by junior staff is more than $50 \%$ of cases delays in providing, experienced staff and timely operations mortality varies between hospitals, the mortality was significantly higher for blunt trauma than in US. This study also recommended early involvement of senior member of the staff. Objective of the study is to assess trend in Trauma care in the United Kingdom

\section{Method}

We have literature search and studies done in the heading of Trauma, emergency services reorganization between 1990 - 2015. A bibliographic search of PubMed, MEDLINE, Health STAR, and CINAHL. Additional manuscripts were identified in the references of reviewed manuscripts; electronic database was searched for journal articles published in the period, January 1990 to December 2015. The search identified the term "Trauma, emergency services reorganization" in the title and/or abstract of a paper, excluding papers not written in English. Research reports, trial protocols, systematic reviews, metaanalyses, discussion pieces, published oral presentations. The search was undertaken systematically. The process of analysing the papers' content and identifying challenges described by authors produced a number of themes which we used to structure the results.

Inclusion criteria were based on methodological criteria (i.e., a comparative \{Controlled\} study). Authors assessed independently strength of evidence demonstrated by each article.

\section{$\underline{\text { Results }}$}

The search resulted in a sample of 1217 studies in the initial electronic data search, following screening of Titles and abstracts, 78 references were identified as potentially relevant to our study. After reviewing abstracts 54 articles were assigned for further analysis, 24 excluded because their subject matter was not relevant for our purpose, on the basis of mixed population of very sick patients with and without trauma (Figure 1).

\section{Discussion}

Trauma care changed in February 1976 when a tragedy occurred, Dr. Jim Styner, an orthopaedic surgeon was piloting his plane and crashed in a rural Nebraska Cornfield. According to Nichol and Turner [7] the current provision of trauma care in the UK is insufficient, resulting high mortality of trauma patients. An audit by National Confidential Enquiry of perioperative death [8] reviewed 795 cases in 2006, which showed that almost 60 percent of the patients received a standard care that was defined as less than good practice the difficulties

Correspondence to: Chandio A, Department of Surgery, Tameside NHS Foundation Trust Hospital, UK, E-mail: chandioashfaq@yahoo.com

Key words: reorganisation of trauma, emergency surgery

Received: September 13, 2016; Accepted: October 16, 2016; Published: October 19,2016 


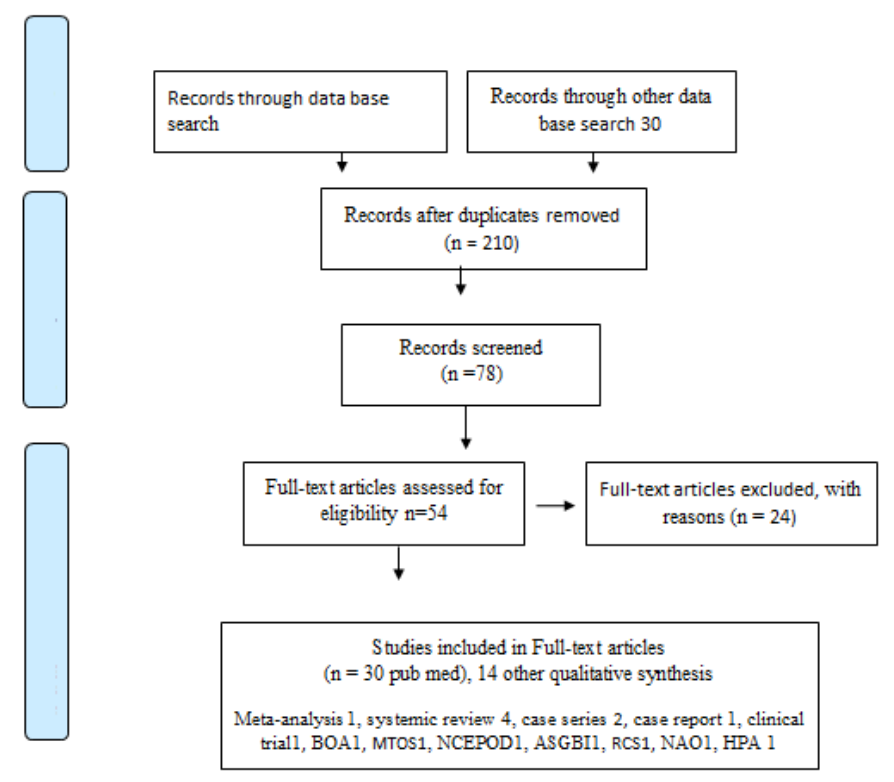

Figure 1. Trauma, emergency services reorganization.

in trauma care are linked to resources. According to the Health Protection Agency [9] in UK 17,000 death each year are a direct result of injuries. The advances in surgery is making almost impossible to provide state of art facilities in all hospitals across NHS. In order to maintain the high standards of care current reconfiguration is essential in NHS as without reconfiguration there would be a rapid decline of quality of care provided in trauma and Emergency General Surgery patients [10]. In opposition to this view [11] from medical care research unit, university of Sheffield have raised concern that reconfiguration could lead to acutely ill patients having to be transported greater distance to the hospital with an associated risk of increased mortality. In 2007 national confidential enquiry of perioperative deaths [8] found deficiencies in both organisational and clinical aspect of trauma care, less than good care for $60 \%$ of reviewed major trauma patients. NCEPOD report recommended need for designated level 1 trauma centres ensuring that a trauma team is available 24/7. Lord Darzi London report in 2007 identified same inequalities in health outcome and the quality and safety of patient care not as good as it could be, his report recommended use of bypass protocols for the most severely injured patients. In order to implement the changes in management of trauma department of health UK appointed a national clinical director for trauma care for the first time on the advice of Professor Keith Willet 2009 this reflects the higher level of priority given to decision making and co-ordination and appointment of local trauma leaders with administration and representation at all levels. Major trauma is serious injury and generally includes injuries like traumatic injury requiring amputation of limb, severe head injury, multiple injuries to chest and abdomen, spinal injury and severe burns according to the Association of Surgeons of Great Britain and Ireland [12]. Emergency general surgery (EGS) is a large and essential service which is the core activity for many acute hospitals typically representing 50\% of all general surgical activity. According to Royal College Of Surgeons England the standards of management of unplanned surgical patients are very variable and often sub-optimal [13]. The management of EGS patients is inefficient due to lack of dedicated acute beds and lack of continuity with the sub-specialisation or rationalisation of EGS, it is essential that receiving hospitals have sufficient capacity to accept referred patients without delay. The issues in management are linked to hours of work, training of staff, experience, elective work load pressure, subspecialisation, targets in other departments (four hour wait in $\mathrm{A} / \mathrm{E}$ ) elective work load pressures and bed numbers [14] techniques such as resuscitative thoracotomy, damage control laparotomy and rapid control of vascular injury are life threatening conditions [15], yet general surgeons working in the UK have few opportunities to develop expertise, increasing number of units expressed interest in dedicated emergency surgeons. Some EG surgeons have adopted successful daily triage role using their experience to assess surgical patients this gives additional exposure to on call surgical team. A regional trauma system is a public health the purpose is to reduce death and disability following injury. According to Brohi et al. [16] the trauma care system improves community health through an organized system of injury prevention, acute care and rehabilitation. Transportation of trauma cases to the appropriate hospital in the network will become effective via appropriate standard ambulance land and air service. According to American college of surgeons committee on trauma [17] an acceptable transportation time within 30-45 minutes is expected to have minimal adverse effect on outcome of trauma. There is current state of under provision of rehabilitation services in NHS [18] a more centralised rehabilitation framework with synchronisation of all available resources is desirable, this model of care seen in many other European countries improves accurate triage and by pass protocols to transfer the patients to major trauma centres are essential. In England, it is estimated that the acute care of major trauma cost the NHS between $£ 0.3$ and $£ 0.4$ billion per year with the loss of annual economic output resulting from major trauma being estimated to be $£ 3.3$ and $£ 3.7$ billion [19]. In US trauma centres are designed level I-IV depending on their capabilities [17]. Level 1 being the highest comprehensive service and Level 4 limited case. According to Kaczynski and Hilton [3] terms of cost per life year saved regionalized trauma centre care costs significantly less than the provision of renal dialysis, breast cancer, surgical management of coronary artery disease and is cost-effective when compared with provision of other medical interventions [3]. According to Goldfarb et al. [20] trauma centre can reduce long term morbidity of survivors, and improves functional outcome, but on the other hand comparison by Goldfarb et al. [20] concluded that level1 trauma centres has relatively high charges per day, and a similar or average longer length of stay than other hospitals American type of system does not appear suitable for the UK due to mainly densely populated areas, shorter transportation distances, presence of infrastructure and role of District general hospitals [11,18]. According to Black [21] similarity exist between Scandinavia and UK with both having large number of smaller hospitals receiving injured patients as seen in UK. National registries have yet to be implemented in Scandinavia in comparison to uk TARN data is one of the main databases in most of the hospitals. According to Gulliver and Simpson [22] injury is the leading cause of death in New Zealanders between ages of 1 and 34 . In comparison to $\mathrm{U} k$ trauma system in New Zealand is different. The trauma centres in New Zealand admit between 100 and 240 patients with injury severity score $>15$. This model of care is different from NHS due to absence of a national trauma system or trauma registry. In the UK, the term major centre (MTC) is used to describe a central hospital as described by Brohi et al. [16]. The MTC is responsible from point of injury until rehabilitation process is complete with such a system an ambulance will bypass small hospitals, if the patient's condition does not permits, and transfer the injured patient to a major trauma centre. Effective systems and job plans are needed to remove the adverse impact of split-site working. UK guideline suggest that systems should be organised so that the MTC manages at least 400 
Cases of major trauma per year and serve a minimum Population of 2-3 million people [16]. The setting up of regional trauma networks is well under way in England. With 4 networks already working in London, and more networks were due to come on line throughout the nation. According to Davenport et al. [23] a comparative analysis of data from the Royal London Hospital (RLH) trauma registry and Trauma Audit and Research Network (England and Wales) 2000-2005 suggest that preventable death rates fell from 9 to 2 percent $(\mathrm{P}=0.040)$. Minor injuries units should have no role in treating severely injured patients; there is need for comprehensive by pass policies in place so that blue light ambulance does not land there. The development of trauma system in the UK lost its progress when the trauma centre pilot study at North Staffordshire Hospital failed to replicate the findings from the United States and summarized that there was no evidence for decreased mortality after the introduction of trauma centre [7].

The limitation of study was due to retrospective data collection, 24 hour consultant cover was limited to accident and emergency only. The recommendation of further pilot programmes to template changes not implemented in UK fully resulting in lack of data, triage issues in pre hospital management. Two strategic health authorities (London and East Midland) have taken a number of steps towards the implementation of regional trauma networks. At the heart of the system would be a major trauma centre equipped to receive and care for the majority of patients at the next level would come trauma units which provide limited and selected trauma care at the third level local hospital would continue to provide existing emergency department services to people with minor injuries. Trauma Audit and Research Network (TARN) is a non-profit organisation (part of University of Manchester). TARN produces monthly and quarterly data of $48 \%$ of acute hospitals in England and TARN does not collect data on trauma patients admitted for less than 3 days. The training of surgeons has suffered after the Implementation of European Working Time directive The European agreement was implemented as law in the UK in 1998. According to Brooks et al. [14] the compressed period of training lead to have difficulty for general Surgeons in techniques such as resuscitative thoracotomy, damage control Laparotomy and rapid control of vascular injuries as lifesaving. The changes to hours of work by junior doctors over the last 20 years have reduced effective exposures to emergency cases, Other than England and Wales there are separate reasons for poor standards of trauma care in Scotland, the geographical distribution of population in Scotland has delayed the introduction of trauma system in Scotland [24]. Data of 141,668 incidents between November 2008 and October 2010 identified $72.1 \%$ occurred in urban region, another difficulty mentioned by Jansen [25] was that the Scottish Government opted against the major trauma care reorganisation the Scottish trauma audit group ceased to collect trauma data in 2002. Australian state Victoria shares many of the geographical challenges that Scotland faces, it has similar population to Scotland (5.6 million vs. 5.2 million) in an area three times the size of Scotland. According to meta-analysis by Casco et al. [26] Victorian state trauma system (VSTS) was established in 2000 and has resulted in $80 \%$ of all major trauma being treated at major trauma centre, reduction in mortality, fewer than expected deaths, positive trends in preventable death and reduced length in hospital stay were the outcomes as a result. The incidence of major trauma in England and Wales is thought to be around four patients per million per week [27]. The reconfiguration of emergency services in a limited number of specialist centres could save thousands of lives each year in the UK. The centralisation on the other hand lead to acutely ill patients travelling for longer distances and an associated risk of increased mortality [10] An observational cohort study by Nichol et al. [11] 10,315 patients transported With potentially life threatening conditions (excluding cardiac arrest) by four English ambulance services between 1997-2001 conclude that each additional kilometre is associated with $2 \%$ relative increase in mortality. While on the other end there are limitations due to observational study, confounding results cannot be generalized for patients having less severe illness, another concern raised by that due to centralization of trauma centres staff in nearby hospitals will become de skilled, health care leaders are looking into this potential risk of staff training issue [28]. Different hospitals in NHS range from large teaching hospital to small rural one. Designing systems that can address these specifics will be the key to improving outcome, particularly for emergency general surgery (EGS). Emergencies account for $80-90 \%$ of general surgical deaths. According to the Association of Surgeons Great Britain and Ireland ASGBI [12] trauma is an important but extremely small part of EGS to which regionalisation is currently bringing real risks as well as prospects of improvement. Facilities for emergency surgery are inadequate with serious deficiencies in theatre in $55 \%$ of units and a comprehensive interventional radiology service is available in only $19 \%$ of units. The NHS needs a frame work to meet the reality of modern surgery. The requirements are to train more surgeons, infrastructure support from the management and to integrate IT facilities for all areas of patient care. According to Nathens [29] for a hospital to develop and maintain optimal skills in major trauma it would need to see 650 cases per year. It is however unlikely that any hospital in England will see this many trauma patients in a year and therefore focus need to be on integrated services between hospitals. In order to maintain the continuity of care for EGS services different models have been designed to deliver the service. A common feature of most is the surgeon of the week where the on call consultant and team are free from all elective responsibilities and available solely to attend to EGS, $10 \%$ of units have adopted a split between upper and lower GI this division will not be applicable in smaller hospitals where there might be more lower GI than upper GI surgeons, this split of work has caused further controversies when an upper GI consultant is not expected to do a low risk colonic resection, but can crack on with a high risk emergency malignant bowel obstruction or take back a rectal anastomosis leak. Suggested division of emergencies between UGI and LGI teams would not be practical in smaller hospitals. With sub-specialisation or rationalisation of EGS, it is essential that receiving hospital have sufficient capacity to accept referred patients without delay much of the research on trauma care in the UK has been carried out using data from the Trauma Audit and Research Network (TARN). The health care commission is working with TARN to increase participation from $50 \%$ to $100 \%$, and to expand the number of quantitative trauma audits. The trauma network database is an important source of epidemiological data and in 2000 it contains information on over 120,000 cases. In isolated areas of Scotland the introduction of a training scheme in remote and rural surgery through attachments is due to bring improvement in morbidity and mortality [30]. The implementation of a trauma system is associated with a 15$50 \%$ reduction in mortality and a reduction in preventable death to close to zero. According to Royal College of Surgeons [31] the mortality percentage is reduced from $19 \%$ to $12 \%$.

\section{Conclusion}

The redesign and reconfiguration process must respect the access need of patients and relatives, well-co-ordinated surgical care to rehabilitation and home care, monitoring of outcome data, assessment of standards through audit process, and the better organisation depends on planning of integrated systems for trauma management 
verification of trauma services through hospitals inspections. A robust clinical governance programme can achieve significant improvement with the reorganisation of trauma centre in the NHS, there is growth of sub specialty of EGS. A period of convectional training in general surgery, minimally invasive approaches, endoscopic skills, and time spent in critical care environment can train future teams of doctors and have better outcomes, in the process of care and outcome from severe injury. There has been increasing concern in recent times of the threat of chemical, biological, and nuclear terrorist attacks, an operational trauma system, combined with integrated major incident planning should enable an enhanced health care response to such incidents. Primary triage of severe trauma patient to the MTC has the potential to offer significantly better care and outcome by concentrating the most seriously injured patients where the expertise exist to care of them.

\section{References}

1. Mains C, Scarborough K, Bar-Or R, Hawkes A, Huber J, et al. (2009) Staff commitment to trauma care improves mortality and length of stay at a level I trauma center. $J$ Trauma 66: 1315-1320. [Crossref]

2. Murray CJ, Lopez AD (1997) Alternative projections of mortality and disability by cause 1990-2020: Global Burden of Disease Study. Lancet 349: 1498-1504. [Crossref]

3. Kaczynski J, Hilton Joanna (2012) Trauma care centre in uk: past, present, future Journal of perioperative surgery.

4. Anderson ID, Woodford M, de Dombal FT, Irving M (1988) Retrospective study of 1000 deaths from injury in England and Wales. Br Med J (Clin Res Ed) 296: 13051308. [Crossref]

5. British Orthopaedic Association; The management of trauma in Great Britain 1997

6. Yates DW, Woodford M, Hollis S (1992) Preliminary analysis of the care of injured patients in 33 British hospitals: first report of the United Kingdom major trauma outcome study. BMJ 305: 737-740. [Crossref]

7. Nichol j ,Turner j 1997 Effectivness of a regional trauma system in reducing Mortality from major trauma; before and after study. British Medical Journal 315 (7119): 1349-54.

8. Henning DC, Smith JE, Patch D, Lambert AW (2011) A comparison of civilian (National Confidential Enquiry into Patient Outcome and Death) trauma standards with current practice in a deployed field hospital in Afghanistan. Emerg Med J 28: 310-312. [Crossref]

9. Health Protection in the 21st century-Understanding the Burden of Disease; Preparing for the future. Part 10: injuries. Health protection Agency, 2005.

10. Brohi K (2008) Regionalisation of trauma services. London reconfiguration, Royal London Hospital in A trauma system for London, version 1.0, Health care for London.

11. Nicholl J, West J, Goodacre S, Turner J (2007) The relationship between distance to hospital and patient mortality in emergencies: an observational study. Emerg Med J 24: 665-668. [Crossref]

12. Association of Surgeon of Great Britain and Ireland. Emergency General Surgery- The future A consensus Statement.
13. MEnSA (2014) study group, on behalf of Mersey Research Group for Surgery Risk stratification, management and outcomes in emergency general surgical patients in the UK. Eur J Trauma Emerg Surg 40: 617-624. [Crossref]

14. Brooks A, Butcher, Walsh M, lambert A, Browne J, et al. (2002) The experience and training of British General Surgeons in Trauma Surgery for the Abdomen, thorax and major vessels. Ann R College of Surgeons England 84: 409-413.

15. Harris T, Davenport R, Hurst T, Jones J (2012) Improving outcome in severe trauma: trauma systems and initial management: intubation, ventilation and resuscitation. Postgrad Med J 88: 588-594. [Crossref]

16. Brohi K, Par T, Cotas T (2009) Regional Trauma Systems. Interim guidance for commissioners. London; Royal College of Surgeons of England. Pp: 1- 60

17. Sasser SM, Hunt RC, Faul M, Sugerman D, Pearson WS, et al. (2012) Guidelines for field triage of injured patients: recommendations of the National Expert Panel on Field Triage, 2011. MMWR Recomm Rep 61: 1-20. [Crossref]

18. Giannoudis PV (2009) Trauma care in the UK and beyond: what are the issues? Injury 40: 681-682. [Crossref]

19. Morse A, Fisher A, Ross C (2010) Major Trauma Care in England. London; National Audit Office. Pp: 1- 41.

20. Goldfarb MG, Bazzoli GJ, Coffey RM (1996) Trauma systems and the costs of trauma care. Health Serv Res 31: 71-95. [Crossref]

21. Black A (2004) Reconfiguration of surgical, emergency, and trauma services in the United Kingdom. BMJ 328: 178-179. [Crossref]

22. Gulliver PJ, Simpson JC (2007) Injury as a leading cause of death and hospitalisation. Fact sheet 38. Injury Prevention Research Unit.

23. Davenport RA, Tai N, West A, Bouamra O, Aylwin C, et al. (2010) A major trauma centre is a specialty hospital not a hospital of specialties. Br J Surg 97: 109-117. [Crossref]

24. Distribution of trauma incidents in Scotland (2013) Br J Surg: 100351-359.

25. Jansen JO (2010) Regionalisation of trauma services in England \& Wales: implications for Scotland. Surgeon 8: 237-238. [Crossref]

26. Celso B, Tepas J, Langland-Orban B, Pracht E, Papa L, et al. (2006) A Systematic Review and Meta-Analysis Comparing Outcome of Severely Injured Patients Treated in Trauma Centres Following the Establishment of Trauma System. J Trauma 60: 371378. [Crossref]

27. Smith N, Weyman D, Findlay G, Martin I, Carter S, et al. (2009) The management of trauma victims in England and Wales: a study by the National Confidential Enquiry into Patient Outcome and Death. Eur J Cardiothorac Surg 36: 340-343. [Crossref]

28. Blakemore S (2009) Trauma centres: a capital idea. Emerg Nurse 17: 6-7. [Crossref]

29. Nathens AB, Jurkovich GJ, Maier RV, Grossman DC, MacKenzie EJ, et al. (2001) Relationship between trauma center volume and outcomes. JAMA 285: 1164-1171. [Crossref]

30. Sedwick D (2005) Remote area surgery. Edinburgh; Royal College of Physicians of Edinburgh.

31. Commissioners (2009) Royal College of Surgeons of England 2009.

Copyright: (C2016 Chandio A. This is an open-access article distributed under the terms of the Creative Commons Attribution License, which permits unrestricted use, distribution, and reproduction in any medium, provided the original author and source are credited. 